sinusitis presented to Temple Street Emergency Department in September 2018. We carried out a case series detailing their presentations, clinical courses and outcomes, and discussed the identification and management of complications of bacterial sinusitis.

Methods Three cases of complicated sinusitis from September 2018 were selected from the emergency department admissions record. Their medical records including lab results and imaging reports were reviewed and comparisons between the three cases were made.

Results The three cases, all male, were aged 14, 11 and 11. All three had had preceding symptoms of coryza, pyrexia and headache. One developed neck pain and vomiting and was initially thought to have meningitis. CT brain revealed left sided sinusitis with an extradural abscess. The other two patients were initially treated as orbital cellulitis. One was transferred from a peripheral hospital, where he had presented with eye swelling and pain, after he developed diplopia and frontal bone swelling. CT showed pansinusitis with a right sided frontal osteomyelitis and a subperiosteal collection in keeping with Pott's puffy tumour. The other presented with eye swelling and pain; CT showed pansinusitis with an extradural empyema. All three patients had markedly raised inflammatory markers on presentation. All required prolonged courses of IV antibiotics. All three had neurosurgery and ENT involvement, the cases of orbital cellulitis had daily ophthalmology reviews. Two had FESS procedures with drainage of their abscess.

Conclusion These three cases demonstrate the potential for bacterial sinusitis to cause serious complications. It is important for the clinician to bear these complications in mind when evaluating the child with rhinosinusitis and to consider imaging when there is a clinical suspicion. Speciality involvement is vital in the management of complicated sinusitis. There is a role for the development of a national clinical guideline pertaining to the management of bacterial sinusitis in the paediatric population.

\section{P277 THE PREVALENCE OF BACTERIAL INFECTION IN RECENTLY VACCINATED FEBRILE INFANTS}

Ali Raba, Ibraheem Krebit*. Paediatric Emergency Department, Tallaght University Hospital, Dublin, Ireland

\subsection{6/archdischild-2019-epa.627}

Background and aims Evidence is lacking regarding the best approach to evaluating recently vaccinated infants younger than 3 months who present to emergency department (ED) with fever. There have not been any recent published studies assessing the prevalence of bacterial infections in this population. The aim of the present study is to investigate the prevalence of bacterial infections in young infants presenting with fever within 72 hours after vaccination.

Methods We reviewed the electronic medical records of infants aged between 6-12 weeks who presented with a fever $>38 \mathrm{C}$ to paediatric ED, Tallaght University Hospital, Dublin, from January 2018 to December 2018. Febrile infants who recently vaccinated within 72 hours prior to $E D$ presentation were compared with those who did not recently vaccinated. Bacterial infection was diagnosed based on culture results (urine or blood or CSF).
Results A total of 70 infants (age: $8.8 \pm 1.9$ weeks, male: 40 $(57.1 \%))$ were enrolled in this study. Among 70 infants, 19 $(27.1 \%)$ had recently vaccinated, of whom $11(57.9 \%)$ presented to ED within 24 hours of vaccination, 7 (36.8\%) presented 24-48 hours after vaccination and 1(5.3\%) presented 48-72 hours after vaccination. The prevalence of bacterial infection in non-recently vaccinated infants was $17.6 \%$ compared to $10.5 \%$ in recently vaccinated infants. Interestingly, all vaccinated infants who had bacterial infections were presented to ED with fever within 24 hours of vaccination and all bacterial infections in this group were urinary tract infection (UTI).

Conclusion Fever should not be attributed only to the vaccinations in young Infants who present to paediatric ED with fever after recent vaccination. All febrile recently vaccinated infants should be carefully evaluated and at least urine testing should be done regardless of the time of vaccination after the presentation.

\section{P278 IMPROVING THE QUALITY OF ACUTE AND INPATIENT MANAGEMENT OF PAEDIATRIC ANAPHYLAXIS THROUGH THE SIMULATION OF INTRAMUSCULAR ADRENALINE ADMINISTRATION}

Nicholas Richens*, Benjamin Miguras, Fathima Zahir. Sandwell and West Birmingham Hospitals NHS Trust, Birmingham, UK

\subsection{6/archdischild-2019-epa.628}

Background The use of intramuscular adrenaline in the acute treatment of paediatric anaphylaxis is well-established and the effectiveness of adrenaline administration is time-dependent. The incidence of anaphylaxis presentations to paediatric emergency departments is increasing, as is the use of adrenaline autoinjectors in the pre-hospital setting. Cost and supply challenges have reduced UK inpatient adrenaline autoinjector usage without evidenced complication and this has resulted in alternative methods of intramuscular adrenaline administration in the acute setting.

Methods In this study we compare the time intervals between anaphylaxis diagnosis and IM adrenaline administration in a low-fidelity simulation of a biphasic type 1 hypersensitivity reaction, repeated across two UK paediatric centres. Time from diagnosis to administration were compared for existing adrenaline emergency kits and adrenaline autoinjectors placed in resuscitation trolleys, the method being alternated as a control for the second reaction phase. Performance was compared across locations stocking paediatric resuscitation equipment where paediatric anaphylaxis presentations were considered most likely. This included two paediatric emergency departments, two paediatric admission units, an allergy investigation unit, and a paediatric inpatient ward. Additional data was obtained on nursing staff satisfaction with these methods and their familiarity with the preparation of intramuscular adrenaline injections.

Results This study demonstrated significantly $(\mathrm{p}<0.05, \mathrm{n}=24$ trials) prolonged administration times using adrenaline emergency kits. Causes for this delay included time required to access controlled-drug storage units, time required to obtain injection equipment, and staff familiarity with the preparation of IM adrenaline syringes. In addition, autoinjector usage of adrenaline was universally favoured by clinical staff for use in the emergency setting. 